\title{
The ionized gas in and around the Wolf-Rayet galaxy NGC 1741
}

\author{
Ángel R. López-Sánchez, César Esteban \\ Instituto de Astrofísica de Canarias, \\ C/ Vía Láctea s/n, E-38200 La Laguna, Tenerife, España \\ Mónica Rodríguez \\ Instituto Nacional de Astronomía, Óptica y Electrónica, \\ Apdo. Postal 51 y 216, 72000 Puebla, Pue., México
}

\begin{abstract}
Interaction can be the main mechanism that triggers star formation in Wolf-Rayet galaxies. The compact group HCG 31 has one of this objects, NGC 1741. We present new observations of this compact interacting group, in order to analyse the physical conditions and chemical composition of the ionized gas, as well as to estimate the ages of the starbursts.
\end{abstract}

\section{Discussion}

The Hickson Compact Group 31 (HCG 31) lies at 51 Mpc (Vacca \& Conti 1992). Galaxies A and C form NGC 1741, which is one of the most luminous WolfRayet galaxies known $\left(\mathrm{M}_{B}=-20.3\right)$. Both objects are in interaction (Johnson et al. 1999), and they host a burst of star formation of around $5 \mathrm{Myr}$ (Johnson \& Conti 2000). Object D is a background galaxy (Rubin et al. 1990). E and F may be tidal dwarf galaxies (TDGs), and F shows a starburst of around $4 \mathrm{Myr}$ (Johnson \& Conti 2000). This last object does not possess an old population. We have distinguished the F1 and F2 zones inside $F$ and the new member $H$. Images showing the location of $F_{1}, F_{2}$ and $H$ will be published elsewhere.

We have obtained long-slit intermediate-resolution spectroscopy. We observe the [O III] $4363 \AA$ emission line in most of the members, and we have been able to derive the electron temperature. Electron densities are always below the low density limit $\left(<100 \mathrm{~cm}^{-3}\right)$, except in member $\mathrm{C}$. For A and $\mathrm{H}$ we have estimated the electron temperature making use of empirical methods. We have also determined the radial velocity of each member. H belongs to HCG 31 because it has a radial velocity similar to the system.

We have determined $\mathrm{O}^{+}, \mathrm{O}^{++}$and $\mathrm{N}^{+}$abundances, as well the $\mathrm{O} / \mathrm{H}$ ratio. We checked the obtained results with empirical calibrations. According to the $\mathrm{O} / \mathrm{H}$ ratio of each object, we can see that there are important differences. Member $\mathrm{C}$ shows the highest abundance (8.55) and $\mathrm{F} 1$ and $\mathrm{F} 2$, the youngest bursts (see below), show the lowest values (8.06 and 8.05). This difference makes it difficult that $\mathrm{F}$ can be considered as a TDG produced from material stripped from $\mathrm{C}$ or $\mathrm{A}$. In fact, the $\mathrm{O} / \mathrm{H}$ ratio of $\mathrm{F}$ is more similar to that of $\mathrm{G}$, or even $\mathrm{B}$. 
Table 1. Abundance ratios and age estimations of HCG 31. For A and H abundances were calculated by empirical calibrations. All the ages are in Myr.

\begin{tabular}{lccccccccl}
\hline \hline member & $\mathrm{A}$ & $\mathrm{B}$ & $\mathrm{C}$ & $\mathrm{E}$ & $\mathrm{F} 1$ & $\mathrm{~F} 2$ & $\mathrm{G}$ & $\mathrm{H}$ & ref. \\
\hline $12+\log (\mathrm{O} / \mathrm{H})$ & 8.45 & 8.31 & 8.55 & 8.29 & 8.06 & 8.05 & 8.26 & 8.40 & this w. \\
$\log \left(\mathrm{N}^{+} / \mathrm{O}^{+}\right)$ & -1.29 & -1.45 & -1.62 & -1.36 & -1.27 & -1.46 & -1.38 & -1.36 & this w. \\
\hline \hline age indicator & & & & & & & & & \\
\hline$W_{\mathrm{H} \beta}$ SL96 & $4-8$ & $6-8$ & $4-6$ & $6-8$ & $2-3$ & $2-3$ & $4-7$ & $4-6$ & this w. \\
$W_{\mathrm{H} \beta}$ SV98 & $5-6$ & $5.5-7$ & $3-4.5$ & $5-6$ & $2-3$ & $2-3$ & $4.5-6$ & $4-5$ & this w. \\
$W_{[\mathrm{O} \text { min] }}$ SL96 & $5-8$ & $5-8$ & $3-5$ & $5-8$ & $2-3$ & $2-3$ & $3-7$ & $3-7$ & this w. \\
$\mathrm{H} \alpha$ and U & $3-10$ & $\sim 10$ & $3-10$ & $\sim 10$ & $3-10$ & - & $\sim 5$ & - & IV97 \\
SSCs photometry & $\sim 5$ & $5-10$ & $\sim 5$ & $1-3$ & $<4$ & - & $\sim 5$ & - & JC00 \\
\hline \hline
\end{tabular}

Another interesting result is the detection of C II $4267 \AA$ in the spectrum of C. This is the first time this recombination line is reported in an $\mathbf{H}$ II galaxy.

We have used the models of Stasińska \& Leitherer (1996) - SL96 in Table 1 - to estimate the age of the bursts. Our observations fit well with a $\mathrm{Z} / \mathrm{Z}_{\odot} \approx 0.25$ and $\mathrm{M} / \mathrm{M}_{\odot} \approx 1000$ burst. We can obtain a good estimation of the age of the bursts plotting the observed $W_{\mathrm{H} \beta}$. We observed that $\mathrm{F} 1$ and $\mathrm{F} 2$ are the youngest members, and we find that they have an age between 2 and $3 \mathrm{Myr}$. The rest of the bursts are more evolved, and they have an age between 4 and $8 \mathrm{Myr}$. We have also used the Schaerer \& Vacca (1998) models - SV98 in Table 1 - to estimate ages, and they provided similar results. We show them in Table 1 as well as other estimations of the age of the burst in the literature.

We can observe clearly the WR-bump between 4650 and $4698 \AA$ in member C. Furthermore, we detect the nebular He II $4686 \AA$ emission in F1. Galaxies A and $\mathrm{B}$ could also show a faint WR-bump. In addition, faint He II $4686 \AA$ emission could be present in F2.

\section{Conclusions}

Deep intermediate-resolution spectra of the different members of HCG 31 show that there are important differences in their chemical content. Member $\mathrm{C}$ shows the highest $\mathrm{O} / \mathrm{H}$ ratio and the youngest burst, $\mathrm{F}$ (age between 2 and $3 \mathrm{Myr}$ ), shows the lowest one. This suggests that $\mathrm{F}$ is not a tidal dwarf galaxy (TDG) produced from material stripped from $C$, instead it can be originated from material from $\mathrm{G}$ or B. We have detected He II $4686 \AA$ emission in F, indicating the presence of Wolf-Rayet stars in this interesting and extremely young object.

\section{References}

Iglesias-Páramo, J., Víchez, J.M. 1997, AJ 479, 190

Johnson, K.E., Vacca, W.D., Leitherer, C., Conti, P.S., Lipscy, S. 1999, AJ 117, 1708

Johnson, K.E., Conti, P.S. 2000, AJ 119, 2146

Rubin, V.C., Hunter, D.A., Ford, W.K. 1990, ApJ 365, 86

Schaerer, D., Vacca, W.D. 1998, AJ 497, 618

Stasińska, G., Leitherer, C. 1996, Ap\&SS 107, 661

Vacca, W.D., Conti, P.S. 1992, ApJ 401, 543 